\title{
Die Schema-Theorie in der Medienwirkungs- forschung: Ein unscharfer Blick in die „Black Box“?
}

\author{
Jörg Matthes
}

Die Schema-Theorie gilt als bewäbrtes Erklärungsmodell in der kognitiv orientierten Medienwirkungsforschung. Gemäß dieser Theorie bängen die Aufnabme und Verarbeitung von Informationen von erlernten, relativ stabilen kognitiven Wissensstrukturen, so genannten Schemata, ab. Ziel dieses Aufsatzes ist eine Bestandsaufnahme der SchemaTheorie in der Medienwirkungsforschung. Nach einem Überblick über die wichtigsten Grundannahmen widmet sich der Beitrag vor allem einer kritischen Betrachtung der Theorie. Es wird argumentiert, dass die Schema-Theorie nicht nur ein vereinfachtes Bild von Informationsverarbeitungsprozessen zeichnet, sondern auch für eine reliable und valide empirische Umsetzung unzureichend ist. Zudem können durch die Schema-Theorie Wirkungsprozesse nur schwer präzise vorhergesagt werden. Anschließend werden daher drei mögliche Alternativen zur Schema-Theorie skizziert: die nicht-strukturelle Schema-Theorie, konnektionistische Ansätze und Einstellungstheorien. Der Beitrag schließt mit einem Plädoyer für eine stärkere Orientierung an aktuellen kognitions- und sozialpsychologischen Konzepten.

Keywords: Schema-Theorie, Schemata, Medienwirkungsforschung, Wissen, Informationsverarbeitung, Konnektionismus, Einstellung

\section{Einleitung}

Seit der Einführung schema-theoretischer Überlegungen in die Medienwirkungsforschung gegen Mitte der 80er Jahre gilt die Schema-Theorie als bewährtes und durchaus beliebtes Modell. Schema-theoretische Argumentationen finden sich bis heute in zahlreichen kommunikationswissenschaftlichen Forschungsfeldern, so in der Nachrichtenforschung (u. a. Graber, 1988; Ruhrmann, 1989), in der Forschung zu Genres und Gattungen (z. B. Gehrau, 2003), in der kognitiven Filmpsychologie (z. B. Ohler \& Nieding, 1996), im dynamisch-transaktionalen Ansatz (Früh, 1996) sowie auch in der AgendaSetting Forschung (z. B. Rössler, 1997; Wolling, Wünsch \& Gehrau, 1998) und in der kommunikationswissenschaftlichen Framing-Forschung (z. B. Scheufele, 2003). Gemäß der Schema-Theorie hängen die Aufnahme und Verarbeitung von Informationen von erlernten, relativ stabilen kognitiven Wissensstrukturen, so genannten Schemata, ab (vgl. u. a. Fiske \& Taylor, 1991). Übertragen auf die kognitive Medienwirkungsforschung ist vor allem relevant, welche Schemata die Rezipienten im Verlauf des Rezeptionsprozesses verwenden und wie sich dadurch Medienwirkungen vorhersagen lassen. Für diese Fragestellungen gilt die Schema-Theorie als etabliertes Erklärungsmuster. Wenn man die Breite und die Häufigkeit ihrer Verwendung betrachtet, ist sie aus der Medienwirkungsforschung eigentlich nicht mehr wegzudenken (vgl. Brosius, 1991; Biocca, 1991; Entman, 1989; Früh, 1996; Hannover, Mauch \& Leffelsend, 2004; Kepplinger \& Daschmann, 1997; Ruhrmann, 1989; Wicks, 1992; Winterhoff-Spurk, 1989).

Ziel des Beitrages ist eine Bestandsaufnahme der Schema-Theorie in der Medienwirkungsforschung. Zwar wurden in der Kommunikationswissenschaft schon vereinzelt Übersichtsarbeiten zur Schema-Theorie vorgelegt (Brosius, 1991; Schenk, 2002, S. 269-196; Wicks, 1992), allerdings erscheint eine erneute Bestandsaufnahme aus drei 
Gründen relevant: Zum Ersten beschäftigen sich die vorliegenden Übersichten zumeist lediglich mit den wichtigsten Grundannahmen der Schema-Theorie und deren allgemeiner Übertragung auf kommunikationswissenschaftliche Fragestellungen. Eine genaue Systematisierung des prognostischen Wertes und der Erfassung von Schemata ist allerdings nur unzureichend erfolgt. Zum Zweiten werden in den bisherigen Übersichten die generellen Kritikpunkte an der Schema-Theorie lediglich erwähnt oder skizziert. Der kritische theoretische Status, den man der Theorie - wie zu zeigen sein wird - bescheinigen muss, wurde damit nicht vermittelt. Zum Dritten wurde nur ungenügend elaboriert, welche Alternativen zur Schema-Theorie zur Verfügung stehen. In diesem Beitrag steht demnach eine kritische Diskussion schema-theoretischer Modelle im Vordergrund. In einem ersten Schritt wird die Schema-Theorie in ihren Grundzügen beschrieben (Kap. 2). Anschließend erfolgt eine Systematisierung schema-theoretischer Anwendungen in der Medienwirkungsforschung. Hier werden ihr Erklärungswert sowie verschiedene Operationalisierungsmöglichkeiten herausgearbeitet (Kap. 3). Darauf folgt eine kritische Betrachtung der Schema-Theorie (Kap. 4). Es wird argumentiert, dass die Schema-Theorie nicht nur ein unzureichendes Bild von Informationsverarbeitungsprozessen zeichnet, sondern auch durch ihre unscharfe und damit schwer operationalisierbare Formulierung keine dezidierten Vorhersagen über Medienwirkungsprozesse erlaubt. Neben der kritischen Reflexion zielt der Beitrag aber auch auf eine Wiederbelebung der Diskussion über diese Theorie in der Medienwirkungsforschung. Abschließend sollen daher die Weiterentwicklung der und mögliche Alternativen zur Schema-Theorie skizziert werden (Kap. 5).

\section{Definition und Funktion von Schemata}

Der Begriff „Schema“ wurde durch die Studien von Bartlett (1932) in die psychologische Forschung eingeführt. Bartlett untersuchte die Erinnerungsleistung von Versuchspersonen bei der Reproduktion einer indianischen Volkssage. Dabei stellte er fest, dass die Versuchspersonen zahlreiche Einzelheiten wegließen und stattdessen die Sage gemäß ihrer eigenen Erwartungen modifizierten. Aus diesen Ergebnissen schloss Bartlett auf generische Wissensstrukturen, so genannte Schemata, die für die „Fehler“ in der Reproduktion verantwortlich sind. Aufgegriffen wurde die Idee erst wieder in den 70er und frühen 80er Jahren, u. a. durch den Computerwissenschaftler Minsky (1975), den Kognitionspsychologen Rumelhart (1980) oder die Sozialpsychologin Markus (1977). Die Schema-Theorie fand daraufhin in vielen Teilgebieten der Psychologie großen Anklang (Fiske \& Linville, 1980). Allerdings lassen sich in der moderneren psychologischen Forschung nur noch selten genuin schema-theoretische Arbeiten finden. ${ }^{1}$

Schemata können folgendermaßen definiert werden: „A schema is a structured cluster of concepts; usually, it involves generic knowledge and may be used to represent events, sequences of events, percepts, situations, relations, and even objects“ (Eysenck \& Keane, 2002, S. 252). Vereinfacht ausgedrückt ist menschliches Wissen gemäß der SchemaTheorie ähnlich wie in einem Schubladensystem organisiert: Prinzipiell gibt es unendlich viele Schubladen, da es ja für jede Situation, jedes Objekt etc. ein Schema gibt. Im

1 Dies verdeutlicht eine Recherche in der Fachdatenbank PsychINFO: Von 1970-1980 lassen sich 10 Fachzeitschriftenaufsätze finden, die den Begriff Schema-Theorie im Titel führen. Während es von 1981 bis 199138 Aufsätze sind, sinkt die Zahl in den Jahren von 1992 bis 2002 auf 16 Aufsätze. Im Jahr 2003 gibt es gar keine derartigen Arbeiten. 
Prozess der Informationsverarbeitung wird entweder eine Schublade geöffnet - was wiederum zum Öffnen von verknüpften Schubladen führen kann - oder alle Schubladen bleiben geschlossen, d. h. die Information wird nicht verstanden. In diesem Fall können auch neue Schubladen gebildet werden. Schemata sind also vorstrukturierte, relativ stabile Wissenspakete, die entweder vollständig aktiviert oder nicht aktiviert werden. ${ }^{2}$ Schemata sind an zwei Stellen des Informationsverarbeitungsprozesses relevant (vgl. im Folgenden Rumelhart, 1980; Taylor \& Crocker, 1981): Trifft eine Information auf das Informationsverarbeitungssystem, wird zunächst das Schema identifiziert, welches am besten auf die einströmende Information passt. Diese Phase der Schema-Identifikation kann als datengeleitet (bottom up) bezeichnet werden. Welches Schema identifiziert wird, bestimmt, ob und wie diese Information verstanden und eingeordnet wird. Des Weiteren steuert ein einmal identifiziertes Schema die Verarbeitung der kommenden Information und auch die Aktivierung von verknüpften Schemata. Diese Phase entspricht der konzeptgesteuerten Informationsverarbeitung (top down) und macht den eigentlichen Kernbereich der Schema-Theorie aus (vgl. Schwarz, 1985). Ferner weisen Schemata eine pyramidale Struktur auf und sind untereinander durch ein Netz von Assoziationen verbunden (Fiske \& Taylor, 1991; Rumelhart, 1980; Taylor \& Crocker, 1981).

Schemata haben drei eng verknüpfte Funktionen: Erstens entlasten sie das Informationsverarbeitungssystem (Entlastungsfunktion), da durch den Rückgriff auf ein Schema nicht jeder Stimulus neu und umfassend verarbeitet werden muss. Eine zweite Funktion besteht in der Strukturierung von Erfahrungen (Strukturierungsfunktion) bzw. in der Bedeutungszuweisung von eintreffenden Informationen: „[W] hen a stimulus configuration is matched against a schema, elements in the configuration come to be ordered in a manner that reflects the structure of the schema" (Taylor \& Crocker, 1981, S. 97). Diese strukturierende Funktion ist die Basis für schema-induzierte Erinnerungsleistungen, denn schema-relevante Informationen werden einfacher und schneller erinnert als schema-irrelevante Informationen (Fiske \& Taylor, 1991; Taylor \& Crocker, 1981). Schemata sind aber nicht nur für „Erinnerungslücken“ verantwortlich sondern auch für Ergänzungen. Personen fügen systematisch Informationen hinzu, die nicht Teil des ursprünglichen Stimulus sind. Minsky (1975) führt in diesem Zusammenhang den Begriff der Standardwerte (default options) ein. Ist beim Abgleich von Schema und Stimulus ein schema-konstituierendes Element nicht im Stimulus vorhanden, führt dies nicht notwendigerweise zum „Misfit“, sondern es werden Standardwerte eingesetzt, wie sie in ähnlichen Situationen vorkommen. Würde man beispielsweise einer Versuchsperson einen Arzt beschreiben und die Person anschließend bitten, die Beschreibung wiederzugeben, könnte es sein, dass die Versuchsperson einen weißen Kittel erwähnt, obwohl dieser nicht Teil der ursprünglichen Beschreibung war. Derartige Ergänzungen ermöglichen eine sinnvolle Kontextualisierung von Informationen. Dies ist die dritte Funktion von Schemata, die Ergänzungsfunktion. Darüber hinaus bilden Schemata die Basis für Problemlösungen und Handlungen.

Da Schemata relativ stabil sind, stellt sich die Frage, wie sie überhaupt entstehen und vor allem, wie sie sich verändern können. Rumelhart (1980) und Rumelhart \& Norman (1978) schlagen hierfür drei Prozesse vor: accretion, tuning und restructuring. Accretion

2 Diese Auffassung stammt aus der frühen Computerwissenschaft. Man ging davon aus, dass menschliches Denken ähnlich wie ein Computer funktioniert: Wissen ist in Dateien organisiert, die gespeichert und geöffnet werden können (Nassaji, 2002). 
bezeichnet das sukzessive Ansammeln von Faktenwissen, z. B. beim Lernen von Telefonnummern oder Namen. Neue Informationen werden zu einem bereits bestehenden Schema hinzugefügt, ohne dass es zu strukturellen Veränderungen in der Wissensorganisation kommt. Wenn allerdings kein Schema für die neue Information herangezogen werden kann, dann ist Lernen durch accretion nicht mehr effektiv. In diesem Fall muss entweder ein bereits bestehendes Schema modifiziert werden (tuning) oder es wird ein neues Schema gebildet (restructuring). Tuning kann auf drei verschiedene Arten erfolgen: Zum Ersten kann durch die mehrfache erfolgreiche Anwendung eines Schemas auf eine Situation das Schema stärker an die Gesamtpopulation der betroffenen Situationen angepasst werden. Zum Zweiten kann ein Schema auf neue Situationen oder Stimuli generalisiert werden, indem ein neuer Aspekt zu dem Schema hinzugefügt wird. Im Gegensatz zu dieser Art des tunings kann zum Dritten auch die Anwendung eines Schemas wiederum nur auf ganz bestimmte Situationen beschränkt werden. Die letzte Form der Schema-Veränderung, das restructuring, bezieht sich auf die Entstehung von neuen Schemata. Hierfür schlagen die Autoren wiederum zwei Prozesse vor: patterned recognition und schema induction. Zunächst kann durch Analogie-Lernen ein neues Schema aus einem bereits bestehenden entstehen (patterned recognition). Beim Prozess der schema induction wird hingegen ein neues Schema gebildet, wenn wiederholt eine vorher unbekannte Stimuluskonfiguration auftritt.

\section{Die Schema-Theorie in der Medienwirkungsforschung}

Wie eingangs erwähnt, findet die Schema-Theorie in vielen Gebieten der Medienwirkungsforschung Anwendung. Es sollen nun aber nur solche Studien diskutiert werden, die sich primär auf schema-theoretische Argumentationen beziehen. Dies sind Arbeiten, die entweder Wirkungsprozesse schema-theoretisch vorhersagen bzw. erklären (prozessorientierte Studien) oder Schemata direkt empirisch erfassen bzw. beschreiben (deskriptive Studien). Arbeiten, die sich lediglich der Schema-Metapher bedienen - wie dies in weiten Teilen der Framing-Forschung der Fall ist (z. B. Pan \& Kosicki, 1993; dazu kritisch Scheufele, 2003) - werden nicht einbezogen. Im Folgenden werden die Studien zweifach systematisiert: Zum einen werden die Arbeiten nach ihrem prognostischen Wert unterschieden, d. h. es wird differenziert, welche Phänomene schema-theoretisch erklärt werden. Zum anderen erfolgt eine Systematisierung nach der Operationalisierung von Schemata.

\subsection{Der prognostische Wert der Schema-Theorie}

Der Erklärungsbeitrag der Schema-Theorie für die Wirkungsforschung lässt sich anhand der oben angeführten drei Hauptfunktionen beschreiben: der Entlastungs-, der Strukturierungs- und der Ergänzungsfunktion. Schließlich wird skizziert, wie sich die Veränderungen von Schemata modellieren lassen.

Schemata erklären zum Ersten, wie die Rezipienten Wissen über Themen, Personen, Objekte oder Sachverhalte verarbeiten bzw. abspeichern (vgl. z. B. Conover \& Feldman, 1984; Fredin \& Tabaczynski, 1993; Miller, Wattenberg \& Malanchuk, 1986; Warlaumont, 1997). Die Organisation menschlichen Wissens anhand von Schemata hat dabei eine entlastende Funktion. Ist ein Schema vorhanden, kann die Information schnell und effizient eingeordnet und verarbeitet werden. Diese Argumentation findet sich beispielsweise in Forschungsarbeiten zur Nachrichtenrezeption: Schemata ermöglichen den Rezipienten, die Nachrichten in einen bedeutungsvollen Kontext zu stellen und da- 
mit schnell zu verstehen. Dadurch kann ein effektiver Umgang mit der Fülle von massenmedial vermittelten Informationen gewährleistet werden (Graber, 1988; Brosius, 1991). Die Schema-Theorie kann darüber hinaus aufzeigen, wie ein Thema von den Rezipienten repräsentiert wird: als kognitives Schema (Eichhorn 1996, S. 81). Ähnlich kann beschrieben werden, welche Schemata die Rezipienten über Wahlkandidaten haben (vgl. Miller et al., 1986). Fernerhin argumentiert die Forschung zu Genres und Gattungen: Genre-, Sender- oder Sendungs-Schemata bestimmen, welche Merkmale ein Format aufweisen muss, um sinnvoll von den Rezipienten eingeordnet zu werden (vgl. Fredin \& Tabaczynski, 1993; Gehrau, 2003; Hannover et al., 2004; Bilandzic, 1999). So beschreibt Bilandzic (1999) die selektive Fernsehnutzung als schema-geleiteten Prozess: Jedes Umschalten wird als neuerlicher Beginn eines Entscheidungsprozesses betrachtet, bei dem ein Genre-, Gattungs-, Themen- oder Sender-Schema aktiviert wird, was dann wiederum zu einer Bewertung des Gezeigten führt. Ist ein Schema für einen Stimulus vorhanden, wird dieser schneller verarbeitet als wenn kein Schema vorhanden wäre (Bilandzic, 1999, S. 97).

Die zweite, eng damit verknüpfte Funktion von Schemata ist die Strukturierungsleistung: Schemata bestimmen, welche Informationen wahrgenommen und erinnert werden (vgl. Coleman, 2003; Garramone, Steele \& Pinkleton, 1991; Graber, 1988; Grimes \& Drechsel, 1996; Prabu, 1996; Rhee \& Capella, 1997; Schwan, 1995; Wicks, 1995). Hiermit kann man beispielsweise erklären, warum Personen bei der Rekonstruktion von Nachrichten systematische Lücken aufweisen: Es werden nur die Details wiedergegeben, die dem initiierten Schema entsprechen. Beispielsweise befragte Graber (1988) in einer viel beachteten qualitativen Studie mehrfach 21 Personen zur politischen Medienberichterstattung und setzte die Aussagen der Personen mit den Medienberichten in Verbindung. Die Autorin konnte zunächst zeigen, dass die Panelteilnehmer nur einen geringen Teil der Medienberichterstattung behalten bzw. dass nur wenige Fakten wiedergegeben werden konnten. Graber führte dieses Ergebnis auf die schema-geleitete Informationsverarbeitung zurück: Es werden die Informationen aus der Medienberichterstattung in bereits bestehende Schemata integriert und damit kontextualisiert. Genauer: Durch die schema-geleitete Informationsverarbeitung verlieren die Informationen ihre Detailhaftigkeit und werden vergleichsweise abstrakter repräsentiert. Ähnlich machte sich auch Schwan (1995) die erinnerungsstützende Funktion von Schemata in einem Experiment zur Filmrezeption zu Nutze: Personen, bei denen durch Vorinformationen ein Genre-Schema aktiviert wurde, konnten sich nach dem Anschauen eines Filmes an mehr Filmszenen erinnern als Personen ohne Vorinformation. Ein analoges Erklärungsmuster findet sich in der Agenda-Setting-Forschung: Vereinfacht ausgedrückt widerspricht die Schema-Theorie einer linearen Abhängigkeit der Publikumsagenda von der Medienagenda. Die Wahrnehmung der Medien-Agenda wird durch bestehende Schemata geprägt, d. h. die Rezipienten verarbeiten neue Informationen selektiv und modifizierend (vgl. Rössler, 1997, S. 395-403).

Die dritte Funktion von Schemata, die Ergänzungsfunktion, kann herangezogen werden, um aktive Konstruktionsprozesse der Rezipienten zu erklären. Diese werden aber in der Medienwirkungsforschung vergleichsweise seltener thematisiert. Fragt man Rezipienten nach dem Inhalt der Medienberichterstattung, dann nennen bzw. ergänzen sie zum Teil Inhalte, die gar nicht in den Medienbeiträgen vorhanden waren. Die Schema-Theorie erklärt das damit, dass nicht alle Elemente eines Schemas vorhanden sein müssen, damit es aktiviert werden kann. Darüber hinaus können sich auch Schemata untereinander initiieren. Ist dies der Fall, so kann ein Schema aktiviert werden, das relativ wenig mit dem medialen Stimulus zu tun hat. Am deutlichsten wurde diese Funktion im 
dynamisch-transaktionalen Ansatz herausgearbeitet (vgl. Früh, 1996). Bei der schematischen Informationsverarbeitung werden Verbindungen zwischen dem medialen Stimulus und bereits vorhandenen Schemata hergestellt. In einer sehr aufwändigen Panelstudie zeigte Früh (1996), wie die Rezipienten die Medienberichterstattung rekonstruieren. Der Autor untersuchte dabei u. a., ob der Medienbeitrag die thematischen Vorstellungsbilder der Rezipienten stärker beeinflusst als dies die subjektiven Schemata tun. Die hierbei zugrunde liegende Logik ist die Folgende: Wenn mehrere Personen dasselbe Thema unabhängig vom medialen Stimulus ähnlich darstellen, dann sind dafür kognitive Schemata verantwortlich (die in diesem Fall bei den Befragten gleich wären). Die Ergebnisse zeigen u. a., dass die kognitive Verarbeitung der medialen Stimuli stärker durch subjektive Schemata beeinflusst werden als durch die medialen Themenstrukturierungen.

Die Frage hingegen, wie sich Schemata verändern, vermögen Studien in der Wirkungsforschung kaum zu beantworten. Einzig Scheufele (2003) hat dies in einer Systematisierung herausgearbeitet. Er unterscheidet drei Fälle, wie Rezipienten-Schemata und mediale Bezugsrahmen zusammenspielen: Im ersten Fall werden durch konsonante und kumulative Berichterstattung die Schemata der Rezipienten sukzessive verändert bzw. der Medienberichterstattung angeglichen. Im zweiten Fall werden Kausalvorstellungen bzw. Attributionen durch die Berichterstattung verschoben. Schließlich erfolgt im dritten Fall eine Etablierung eines neuen Sub-Schemas, wenn noch kein spezifisches Schema für den jeweiligen Gegenstand vorhanden ist. Eine empirische Überprüfung (der Unterscheidbarkeit) dieser Prozesse steht allerdings noch aus.

\subsection{Die Erfassung von Schemata}

In der empirischen Wirkungsforschung werden Schemata mit Befragungen und Experimenten erfasst. Im Folgenden sollen die einzelnen Methoden anhand von Beispielstudien vorgestellt werden:

\section{Befragung}

Die Techniken zur Erfassung von Schemata können vereinfacht in (1) offene Fragen, (2) geschlossene Fragen und (3) Datenreduktionsverfahren eingeteilt werden. Ein Großteil der Studien erfasst Schemata mit qualitativen Techniken wie offenen Fragen oder Tiefeninterviews (vgl. z. B. Garramone et al., 1991; Graber, 1988; Höijer, 1992; Wolling, Wünsch \& Gehrau 1998; Rhee \& Cappella, 1997; Shen, 2004; Warlaumont, 1997). Meist werden die Antworten der Befragten kodiert oder ausgezählt und anschließend entsprechend als Schema interpretiert. Beispielsweise untersucht Warlaumont (1997) die Wahrnehmung von realitätsnahen Werbeformen. In offenen Fragen werden die Reaktionen der Rezipienten auf diese Werbeformen abgebildet. Eine Inhaltsanalyse dieser offenen Fragen konnte zeigen, dass 73 \% der Befragten diese Werbeform so nicht erwartet hatten. Daraus schließt die Autorin, dass realitätsnahe Werbedarstellungen nicht dem gängigen Werbe-Schema der Rezipienten entsprechen: Die erhaltenen Informationen seien schema-inkonsistent.

Eine weitere Möglichkeit, Schemata zu erfassen, sind geschlossene Fragen (vgl. Coleman, 2003; Fredin \& Tabaczynski, 1993; Hamill, Lodge \& Blake, 1985). Dabei werden allerdings häufig klassische Einstellungs- oder Meinungsitems verwendet und deren Ausprägung als Schemata interpretiert. So untersucht Coleman (2003), ob durch die Rasse eines Medienakteurs das ethische Schlussfolgern der Journalisten beeinflusst wird. 
In einem Experiment werden Journalismusstudenten verschiedene Nachrichtentexte vorgelegt und u. a. die Rasse der Akteure manipuliert. Anschließend füllen die Versuchspersonen das standardisierte „Journalists' Ethical Reasoning Instrument“ aus. Die Ergebnisse zeigen signifikant geringere Werte auf der Skala bei afrikanischen im Vergleich zu weißen Amerikanern. Erklärt werden diese Ergebnisse schema-theoretisch: Die Antworten auf die geschlossenen Fragen wertet die Autorin als Aktivierung eines Rassen-Schemas.

Eine letzte Technik stellen die Datenreduktionsverfahren dar: In der Studie von Miller et al. (1986) werden die aus offenen Fragen gebildeten, inhaltsanalytischen Kategorien in Dummy-Variablen aufgespalten und anschließend faktorisiert. ${ }^{3}$ Ein weiteres, seltener angewandtes Verfahren ist die Q-sort Methode (vgl. Bolland, 1985; Conover \& Feldman, 1984). Bei dieser Methode werden die Befragten einzeln gebeten, Stimuli (z. B. Items, Personen) in selbst gewählte Kategorien einzusortieren und zu bewerten. Die Auswertungen erfolgen korrelations- bzw. faktorenanalytisch. In der Studie von Conover \& Feldman (1984) sollte jeder einzelne Befragte Statements aus verschiedenen politischen Themenbereichen in verschiedene Zustimmungskategorien einordnen. Ergebnis dieser Studie sind insgesamt 17 verschiedene Schemata, wie beispielsweise „basic human philosophy“ oder „racial beliefs“.

\section{Experimente}

Die vor allem in der kognitiven Psychologie klassische Methode zur Erfassung von Schemata ist das Experiment. Bei Experimenten werden die Schemata allerdings nicht direkt erfasst, sondern es werden Vorhersagen aus der Schema-Theorie abgeleitet und experimentell überprüft. Stimmen die Vorhersagen mit den empirischen Daten überein, wird auf das Vorhandensein oder Wirken von Schemata geschlossen. Für die Wirkungsforschung sind hier vor allem die Studien von Grimes \& Drechsel (1996), Prabu (1996), Schwan (1995), Wicks \& Drew (1991) und Wicks (1995) zu nennen. Beispielsweise untersuchen Wicks \& Drew (1991) in einem Experiment den Einfluss von schema-konsistenten bzw. inkonsistenten Informationen auf die Erinnerungsleistung. Die Versuchspersonen erhielten zwei Zeitungsartikel, die entweder konsistent oder inkonsistent waren. Gemessen wurde dann die Wirkung von Schemata durch freie Erinnerung, gestützte Erinnerung und das Testen von Schlussfolgerungen. Die Autoren zeigen entgegen der ursprünglichen Annahme der Schema-Theorie (vgl. Kap. 2), dass schemainkonsistente Informationen zu einer höheren Erinnerungsleistung führen als schemakonsistente Informationen. Das heißt, es wird davon ausgegangen, dass nach dem Lesen des ersten Artikels ein Schema aktiviert wurde. Der zweite Artikel beinhaltete dann konsistente oder inkonsistente Informationen zu diesem Schema. Impliziert wird, dass die schlechtere Erinnerungsleistung bei schema-konsistenter Information auf die Wirkung eines Schemas zurückzuführen ist.

3 Von einer explorativen Faktorisierung von nominalen Variablen auf der Basis von PearsonKorrelationen wird allerdings in der methodischen Literatur abgeraten (vgl. Cromrey, 1988, S. 758). 


\section{Kritik an der Schema-Theorie}

Für die Medienwirkungsforschung lassen sich drei zentrale Kritikbereiche ausmachen: (1) Kritik an der definitorischen Präzision, (2) Kritik an der prognostischen Präzision und (3) Kritik an der Operationalisierung von Schemata.

\subsection{Definitorische Präzision}

"[If] one asks the question, do we have a good theory of schemas, the answer is unquestionably no“ (Taylor \& Crocker, 1981, S. 125). Diese immer noch gültige Kritik hängt damit zusammen, dass in vielen schema-theoretischen Studien nicht präzise ersichtlich ist, was überhaupt ein Schema ist (Alba \& Hasher, 1983; Brosius, 1991; Brown, 1979; Herrmann, 1982; Iran-Nejad, 1987; McGraw, 2000; Sadoski, Paivio \& Goetz, 1991; Taylor \& Crocker, 1981; Wicks, 1992). Die Begriffsunklarheit spiegelt sich nicht nur in den zahlreichen synonym verwendeten Begriffen wie Skript, Frame, Kategorie oder Stereotyp wider sondern teilweise auch in den Definitionen selbst. So bezeichnen Tannen und Wallatt (1993, S. 60) Schemata als Erwartungen über Menschen, Objekte, Ereignisse und Gegebenheiten (settings) in der Welt. Warlaumont (1997, S. 39) spricht von „patterns of information“, Markus (1977, S. 64) von kognitiven Generalisierungen und Kepplinger und Daschmann (1997, S. 549) von „internal patterns which guide perception and organize information processing “. Schwarz (1985, S. 273) räumt zudem ein, dass man Schemata auch als Wissen bezeichnen kann, da sie abstrakte Repräsentationen vergangener Erfahrungen sind. Früh (1996, S. 17) spricht ebenso allgemein von einem „Wissenskomplex, der Konzepte über Objekte oder Ereignisse in verallgemeinerter Form enthält". Verwirrend ist auch die Definition Entman’s (1989, S. 349), bei der Schemata gleich mit mehreren Begriffen vermengt werden: „A person’s schema stores substantive beliefs, attitudes, values and preferences“. Bezeichnend für die Begriffsproblematik ist ebenso die Aussage von Crockett (1987, S. 33), Schemata seien besser durch Beispiele als durch abstrakte Definitionen zu vermitteln. So verwundert es kaum, dass Herrmann (1982, S. 6) von einem „begrifflichen Monstrum“ bzw. einer „Begriffsinflation“ (S. 5) spricht, die den Leser mit seinem Wissen über die nicht explizierten Begriffe allein lässt. Schwarz (1985, S. 273) macht darauf aufmerksam, dass häufig jede Art der konzeptgesteuerten Informationsverarbeitung mit schematischer Informationsverarbeitung gleichgesetzt wird. Die implizite Gleichsetzung von Schemata mit Vorwissen erscheint aber für eine präzise Erfassung ungenügend. Ein Grund für diese begriffliche Unschärfe ist sicherlich die Verwendung des Schema-Begriffs in vielen verschiedenen Kontexten und Disziplinen (Taylor \& Crocker, 1981). Ein weiterer Grund kann auch in der recht metaphorischen theoretischen Sprache (z. B. Pakete, Gitter, Muster, lagern, abrufen, abspeichern) ausgemacht werden (Iran-Nejad, 1987; Sadoski et al., 1991). ${ }^{4}$

Ebenso unpräzise wird das Abstraktionsniveau von Schemata festgelegt. Bei den von Bolland (1985) extrahierten Schemata wie "health care“, „economy“ oder „education“ handelt es sich offensichtlich um Themen, in anderen Studien geht es aber zumeist um

4 Der Schema-Begriff ist im Grunde ein Begriff aus der Alltagssprache, der zunächst intuitiv plausibel ist und somit anscheinend wissenschaftlich nicht immer genau expliziert wird. Aus den gängigen nicht-operationalen Definitionen wird nicht ersichtlich, welche Elemente vorhanden sein müssen, damit von einem Schema zu sprechen ist. 
Themenaspekte. Was es mit Schemata wie „basic human philosophy“ oder „racial beliefs" (Conover \& Feldman, 1984) auf sich hat, liegt wahrscheinlich ohnehin im Auge des Betrachters. Folglich fragen Sadoski et al. (1991, S. 466), ob wir ein übergeordnetes Schema für die „Farbe“ oder eher Schemata für einzelne Farben haben oder ob in den Schemata für einzelne Farben auch Beispielobjekte $($ Zitrone $=$ gelb) repräsentiert sind. Zwar sprechen Schema-Theoretiker in diesem Zusammenhang von eingebetteten, verknüpften oder hierarchischen Schemata, für Sadoski et al. (1991, S. 466) ist diese Erklärung allerdings nicht zufrieden stellend: „[T]his approach invites infinite regress and theoretical circularity“. Ähnlich fragen Thorndyke \& Yekovich (1980, S. 41), wie detailliert überhaupt ein Schema abgespeichert wird und wie oft ein Ereignis auftreten muss, damit ein Schema erstellt wird: „Do we also have schemata for ,boarding a city bus' or ,buying a pretzel on the street corner in New York City'?"

\subsection{Prognostische Präzision}

Der erste oben beschriebene (vgl. Kap. 3.1) prognostische Wert von Schemata bezog sich auf die Entlastungsleistung. Allerdings zeichnet die Annahme, dass die Rezipienten für viele Nachrichten, Sendeformen, Personen, Genres, Werbegattungen etc. in ihrem „mentalen Schubladensystem“ vorgefertigte, abrufbare Schemata bereithalten, ein zu einfaches Bild von Informationsverarbeitungsprozessen. Aus der Perspektive der Schema-Theorie geht es nämlich beim Prozess der Informationsaufnahme um die Aktivierung bzw. den Abruf eines vorher „abgespeicherten“ Schemas. Genau eben dies macht den entlastenden Charakter der Schemata ja aus. Ein gesamtes Schema wird entweder aktiviert oder nicht aktiviert. Wird dann ein Schema „gefunden“, wird es in derselben Form abgerufen, in der es vorher abgespeichert wurde - ähnlich einer Datei in einem Computer: „Schemata are data structures for representing the generic concepts stored in memory" (Rumelhart \& Ortony, 1977, S. 101, eigene Hervorhebung; ebenso Neisser, 1967, S. 6-8). Zwar kann sich ein Schema im weiteren Prozess auch ändern, aber selbst das geänderte Schema wird im nächsten Schritt wieder gespeichert und bei der nun darauf folgenden Informationsaufnahme erneut abgerufen. Diese Auffassung von Schemata macht nicht nur einen Grundpfeiler der ursprünglichen Theorie aus (vgl. Rumelhart \& Ortony, 1977, S. 101), sondern sie wird auch in den kommunikationswissenschaftlichen Anwendungen der Schema-Theorie so vertreten (vgl. Bilandzic, 1999, S. 97; Hannover et al, 2004, S. 177; Miller et al., 1986, S. 524; Wicks, 1992, S. 116; Wicks \& Drew, 1991, S. 157). ${ }^{5}$ Schemata werden gewissermaßen als Pakete von Informationen verstanden (z. B. „meaningful packets or bundles“, Warlaumont, 1997, S. 410; ähnlich Fredin \& Tabaczynski, 1993, S. 802). Allein aber schon der Begriff „Schema“ (bzw. Paket oder Bündel) ist zu statisch, um menschliche Informationsverarbeitung adäquat zu beschreiben. Dass eine solche Auffassung von Informationsverarbeitungsprozessen schlicht zu starr und damit unzureichend ist, wird immer wieder von verschiedenen Autoren betont (vgl. Anderson, 1977; Brown, 1979; Edwards \& Middleton, 1987; Iran-Nejad, 1987; Nassaji, 2002; Pospeschill, 2004; Rumelhart, Smolensky, McClelland \& Hinton, 1986;

5 Zwischen einer kommunikationswissenschaftlichen und einer psychologischen Schema-Theorie zu unterscheiden, macht nicht nur vor diesem Hintergrund wenig Sinn. Wenn in der Kommunikationswissenschaft von der Schema-Theorie die Rede ist, dann ist damit immer die ursprüngliche Theorie gemeint, wie sie beispielsweise von Rumelhart (1980) beschrieben wurde. 
Smith, 1996; Wirth, 1997). Es gibt kein Element eines kognitiven Netzwerkes, das nur einem einzigen Schema angehören kann. Das kognitive Netzwerk ist plastischer und dynamischer aufzufassen, es ist ständig in Veränderung. Ähnlich meinte auch Anderson schon im Jahr 1977 (S. 421): „It follows that people do not function by selecting the right template from a great mental warehouse of templates abstracted from prior experience. This process must be more dynamic."

Auch der zweite oben herausgearbeitete prognostische Wert der Schema-Theorie die Strukturierungsleistung - ist zu kritisieren. Gemäß der Schema-Theorie erfolgt die Repräsentation eines komplexen Stimulus unvollständig bzw. ungenau, da nur schemarelevante Informationen wahrgenommen und gespeichert werden. Zudem sei die Erinnerung für schema-konsistente Informationen höher als für schema-inkonsistente. Allerdings zeigen viele Studien, dass Erinnerungen ebenso korrekt und sehr detailliert sein können (vgl. Ost \& Costall, 2002; Locksley, Stangor, Hepburn, Grosovsky \& Hochstrasser, 1984; Wynn \& Logie, 1998; Zuroff, 1989). Dies kann die Schema-Theorie nicht erklären (Wirth, 1997, S. 127). Des Weiteren ist in zahlreichen Studien die Erinnerungsleistung für schema-inkonsistente Informationen sogar weitaus höher als für schema-konsistente Information (vgl. Alba \& Hasher, 1983; Sadoski et al., 1991; Wicks \& Drew, 1991). Hierfür wurden zwar schema-theoretische Modelle erweitert (z. B. Schema-Copy-Plus-Tag-Model, Graesser \& Nakamura, 1982). Die Vorhersage dieser Prozesse bleibt aber dennoch eher vage (Nassaji, 2002). In der Wirkungsforschung wurden schlicht beide Vorhersagen empirisch überprüft und bestätigt (so z. B. Schwan, 1995 und Wicks \& Drew, 1991). Dies erschwert eine Ableitung von konkreten Hypothesen. ${ }^{6}$

Die dritte Funktion von Schemata, die Ergänzungsfunktion, erklärt die oben beschriebenen aktiven Konstruktionsprozesse der Rezipienten. Allerdings kann auch hier die Schema-Theorie keine spezifischen Vorhersagen leisten (Sadoski et al., 1991; Taylor \& Crocker, 1981; Thorndyke \& Yekovich, 1980). Vielmehr lassen sich nahezu alle empirischen Ergebnisse schema-theoretisch deuten: Wird beispielsweise die Medienberichterstattung von den Rezipienten nicht reproduziert, so könnte man dies auf zweierlei Weise erklären: Zum einen ist denkbar, dass die Rezipienten die Information gar nicht wahrgenommen haben, da sie nicht dem ursprünglichen Schema entsprach. Zum anderen wäre ebenso denkbar, dass die Medienberichterstattung zur Initiierung von Schemata geführt hat, die nicht mehr viel mit dem Stimulus zu tun hatten. Dieses Beispiel verdeutlicht die immer wieder kritisierte Flexibilität der Schema-Theorie in Bezug auf die empirischen Ergebnisse: „It can be said, in fact, that schema conceptualizations are not so much serving effect predictions as they are giving a degree of plausibility to post facto accounts of observed media effects" (Zillmann \& Brosius, 2000, S. 38-39; vgl. auch Eysenck \& Keane, 2002; Taylor \& Crocker, 1981; Thorndyke \& Yekovich, 1980). Mit anderen Worten, es mag recht einfach sein, empirische Daten schema-theoretisch zu erklären bzw. aus den Daten Schemata herauszulesen, es ist allerdings recht schwierig, Daten $\mathrm{zu}$ finden, die sich nicht schema-theoretisch erklären lassen. Deutlicher: Die Sche-

6 Damit drängt sich die schon 1980 von Thorndyke und Yekovich gestellte Frage auf, inwieweit der Theoriebegriff für die Schema-Theorie letztlich geeignet ist. Vor allem die auch bei Thorndyke \& Yekovich herausgestellten Kriterien „Vorhersagekraft“ und „Testbarkeit“ legen nahe, dass die Schema-Theorie den Anforderungen an eine wissenschaftliche Theorie nicht wirklich genügt. Aus sprachlichen Gründen soll aber auch im Folgenden an der Bezeichnung SchemaTheorie festgehalten werden. 
ma-Theorie ist derart flexibel, dass sie nicht falsifiziert werden kann. Dies ist für Taylor \& Crocker (1981, S. 126) das verheerendste Manko überhaupt. ${ }^{7}$

Ein weiterer kritischer Punkt ist die mangelnde Aussagekraft der Schema-Theorie zur Entstehung und Veränderung von Schemata (Anderson, 1977; Iran-Nejad \& Winsler, 2000; Nassaji, 2002; Kuklinski, Luskin \& Bolland, 1991; Taylor \& Crocker, 1981; Thorndyke \& Yekovich, 1980). Auch hier erweist sich die Annahme von vorstrukturierten Wissenspaketen als zu statisch. Zwar werden bei Rumelhart \& Norman (1978) sowie Scheufele (2003) Prozesse der Schema-Entstehung und -Veränderung differenziert und auch bei Wolling et al. (1998) und Eichhorn (1996) werden Veränderungen angenommen. Allerdings sind diese eher als Systematisierungen bzw. als unspezifische Vorhersagen zu werten. Die Schema-Theorie ist bisher weder in der Lage gewesen, die Bedingungen für Veränderungen exakt zu formulieren, noch diese einer dezidierten Prüfung zu unterziehen. Die vielmals zitierten Prozesse der Schema-Änderung - subtyping, conversion und bookkeeping - stammen ursprünglich sogar aus Forschungsarbeiten zu sozialen Stereotypen (vgl. Weber \& Crocker, 1983), in denen der Schema-Begriff nicht einmal auftaucht. Ihre Generalisierbarkeit auf andere Wissensformen führt zu begrifflicher Unschärfe und ist empirisch nicht gesichert. Dies wird in vielen Übersichtsarbeiten allzu oft übersehen (z. B. neben vielen anderen Wicks, 1992).

Schließlich ist - auch aus Sicht der Medienwirkungsforschung - die vollkommene Vernachlässigung von affektiven Komponenten diskussionswürdig (Kuklinski et al., 1991; Sadoski et al., 1991; Taber, Lodge \& Glathar, 2001; Wilcox \& Williams, 1990). Die Schema-Theorie macht keine validen Aussagen darüber, wie Kognition und Affekt zusammenhängen. Neuere Arbeiten der Kognitionsforschung zeigen jedoch, dass affektive und kognitive Komponenten nicht zu trennen sind (vgl. Bassili \& Roy, 1998): „[A]ll social stimuli are affect-laden“ (Taber et al., 2001, S. 198). So ist auch bei der Nachrichtenrezeption nicht davon auszugehen, dass die Rezipienten einfach ein rein kognitives Schema abrufen, was erst später als Grundlage für Bewertungen dienen kann. Vielmehr sind die abgerufenen Informationen bereits affektiv „besetzt“ und aktivieren automatisch Bewertungen (Morris, Squires, Taber \& Lodge, 2003). Dies lässt sich leicht an der Berichterstattung über den Irak-Krieg vergegenwärtigen: Die Rezipienten werden wohl kaum ein kognitives „Kriegsschema“ ohne jegliche Bewertung aktivieren. Zwar argumentieren einige Autoren mittlerweile auch mit „affektiven“ Schemata. Inwieweit die Schema-Theorie hiermit den bereits etablierten emotionspsychologischen Konzepten das Wasser reichen kann, muss offen bleiben. Ohnehin ist zu befürchten, dass eine derartige Ausdehnung eher zu weiterer begrifflicher Unschärfe statt zu erhöhtem prognostischen Gehalt führt (Wilcox \& Williams, 1990). Und der Unterschied zum Konkur-

7 Scheufele (2004, S. 34) führt gegen dieses Argument ins Feld, dass der Einwand gleichermaßen für andere Konstrukte wie Einstellungen gelte. Moderne Einstellungstheorien legen allerdings klar falsifizierbare Sätze vor, die - im Gegensatz zur Schema-Theorie - auch weitestgehend widerspruchsfrei bestätigt werden (vgl. u. a. Ostrom, Skowronski \& Nowak, 1994). Zudem wird in der Einstellungsforschung weitaus mehr Gewicht auf die reliable und valide Operationalisierung gelegt (vgl. Ostrom, Bond, Krosnick \& Sedikides, 1994). Dies ist u. a. am weit verbreiteten Einsatz von konfirmatorischen Faktorenanalysen zu erkennen, mit denen Einstellungen bzw. Einstellungsstrukturen nach einheitlichen Richtlinien falsifiziert werden können. Schließlich machen Einstellungstheorien keine operationalen Aussagen über empirisch schwer abbildbare hierarchische Strukturen, wie dies die Schema-Theorie tut. Das Problem, dass nicht klar ist, auf welchem Abstraktionsniveau gemessen werden soll, haben Einstellungstheorien ebenso wenig. 
renzkonstrukt der Einstellung wäre dann auch nicht mehr sichtbar (Kuklinski et al., 1991, S. 1345).

\subsection{Methodische Präzision}

Neben der definitorischen und prognostischen Präzision liegt in der Erfassung von Schemata ein weiteres zentrales Problem. Die schon von Fiske \& Linville (1980) und Taylor \& Crocker (1981) geforderte Präzisierung der empirischen Erfassung steht bis heute noch immer aus (Eveland, Marton \& Seo, 2004).

Wie bereits dargestellt wurde, ist eine weit verbreitete Möglichkeit zur Erfassung von Schemata der Einsatz von offenen Fragen. Bei diesem Vorgehen ist aber nicht klar, wie sich die Schemata strukturell abbilden lassen. So liegt es meist im Ermessen des jeweiligen Forschers, auf welchem Abstraktionsniveau Schemata ausgemacht werden. Zudem unterscheiden sich die Befragten möglicherweise in der Tendenz, ausführlich auf offene Fragen zu antworten, was Konsequenzen für die Interpretation von Schemata nach sich zieht. Gravierender: Bei der Verwendung von offenen Fragen zur SchemaMessung kann jede beliebige Aussage vom Forscher als „Schema“ interpretiert werden. Dies führt dazu, dass in der Forschungsliteratur eine endlose Liste von verschiedenen Schemata identifiziert wurde, ohne zu explizieren, ab wann von einem Schema zu sprechen ist. Überspitzt formuliert: Man bekommt immer ein Schema. Im Vergleich zu den offenen Fragen werden bei geschlossenen zwar die Auswertungsoptionen erhöht, allerdings bleibt ebenso unklar, ob die Schemata in der abgefragten Form tatsächlich bestehen. So messen Fredin \& Tabaczynski (1993) Medien-Schemata mit Fragen wie „The media carry too much bad news about metropolitan Columbus." Wie kann ein Forscher a priori wissen, welche Schemata die Befragten ausgebildet haben? Kuklinski et al. (1991, S. 1344) bringen die Gefahr dieses Vorgehens auf den Punkt: „Different researchers, armed with different ideas and different sets of questionnaire items, will inevitably discover different schemas." Nicht nur bei offenen oder geschlossenen Fragen, sondern auch bei den oben beschriebenen Datenreduktionsverfahren gibt es Operationalisierungsprobleme. Hier bleibt offen, inwieweit sich wirklich an schema-theoretische Prämissen gehalten wird. Die Ergebnisse der Studien von Miller et al. (1986) und Conover \& Feldman (1984) hätten auch ohne eine schema-theoretische Argumentation erfolgen können: „[S]chema theory is not an essential framework for these results" (Wilcox \& Williams, 1990, S. 381). Bei der Q-sort Methode (vgl. Conover \& Feldman, 1984) hingegen werden affektive Kategorien nicht sauber von kognitiven getrennt, wie dies die Schema-Theorie impliziert.

Bei experimentellen Erinnerungstests wird auf die Wirkung eines Schemas geschlossen - das Schema selbst wird in seinem Abstraktionsgrad und in seinem Umfang nicht operationalisiert. Höhere Wiedererkennungsraten für schema-relevante Informationen im Vergleich zu schema-irrelevanten Informationen werden zumeist als Beweis dafür gewertet, dass Schemata die Informationsverarbeitung erleichtern. Allerdings geben Kardash, Royer \& Greene (1988) zu bedenken, dass man von Erinnerungsdaten nicht immer präzise auf den Prozess der Informationsaufnabme schließen kann: „Schema activation can shift the subset of stored material that is the focus of the recall process, but there remains the question as to what was the nature of the total set of information acquired when the text was initially read“ (S. 325). Auch Locksley et al. (1984) merken an, dass Wiedererkennungsmessungen zweideutiger sind, als dies Schema-Theoretiker einräumen würden.

Die Methoden zur Erfassung von Schemata in der Medienwirkungsforschung haben 
mit genau den gleichen Problemen zu kämpfen, die schon seit Jahren in der psychologischen schema-theoretischen Literatur diskutiert bzw. kritisiert werden (Kuklinski et al., 1991; Taylor \& Crocker, 1981). Am problematischsten erscheint aber die am häufigsten verwendete Methode, die offenen Fragen. Diese ermöglichen eine zu freie Interpretation der Ergebnisse ohne Berücksichtigung schema-theoretischer Prämissen. Einen etwas besseren Zugang bieten möglicherweise geschlossene Fragen - gesetzt den Fall, die Schemata werden klar und präzise definiert. Auch Scheufele (2004) hält für frequentierte Themen Item-Batterien für möglich. Allerdings gilt es hier, den Unterschied zu klassischen Meinungs- und Einstellungsitems herauszustellen. Bei Experimenten entsteht wiederum das Problem, dass nahezu alle Ergebnisse auf die Wirkung von Schemata zurückgeführt werden können. Zudem bleibt offen, wie das Abstraktionsniveau von Schemata festzulegen und die hierarchische Struktur abzubilden ist. Grundproblem bei allen Methoden ist die Nicht-Falsifizierbarkeit von Schemata. Zurückzuführen sind all diese Schwierigkeiten auf die unscharfe Definition und Konzeption von Schemata.

\section{Weiterentwicklungen und mögliche Alternativen zur Schema-Theorie}

Es ist nicht von der Hand zu weisen, dass die Schema-Theorie zu einer Fundierung der Wirkungsforschung beigetragen hat. Durch die Schema-Theorie konnten Informationsverarbeitungsprozesse beschrieben werden, die vorher schlicht als „Black Box“ behandelt wurden. Wie mit jeder anderen Theorie auch, gilt somit die Schema-Theorie trotz aller Kritik solange als nützlich, bis nicht andere Ansätze oder Theorien die zu untersuchenden Sachverhalte besser und genauer erklären können. Welche anderen Ansätze und Theorien könnten das aber sein? Gesucht sind Konzepte, die eine stärkere definitorische Klarheit, einen dynamischeren Wissensbegriff, einen höheren prognostischen Gehalt und eine größere methodische Präzision mitbringen. Zunächst einmal bietet es sich an, nach konkreten Weiterentwicklungen der Schema-Theorie Ausschau zu halten, die explizit auf Schwächen der Schema-Theorie eingehen. Hier ist einzig die nicht-strukturelle Schema-Theorie zu nennen. Zum Zweiten ist auf neuere Konzepte aus der kognitiven Psychologie zu verweisen, die ähnlich wie die Schema-Theorie Begriffe wie Wissen, Lernen und Erinnern erklären können, dies aber besser tun als die SchemaTheorie. Ein sehr prominentes Theoriegebäude, das auch in den Sozialwissenschaften immer mehr Aufmerksamkeit erlangt, ist der Konnektionismus. Schließlich gibt es seit der Einführung der Schema-Theorie gewissermaßen Konkurrenzbegriffe, an denen die Schema-Theorie schon immer gemessen wurde. Ein solcher Konkurrenzbegriff ist der Einstellungsbegriff. In den folgenden Abschnitten sollen diese drei Konzepte - die nichtstrukturelle Schema-Theorie, der Konnektionismus und die Einstellungsforschung mit Blick auf die Leistung der Schema-Theorie vergleichend vorgestellt werden. Da es sich zumindest beim Konnektionismus (vgl. Pospeschill, 2004) und bei der Einstellungsforschung (vgl. Ostrom, Skowronski \& Nowak, 1994) um komplexe, viel debattierte und untersuchte Konzepte handelt, können diese hier nur in ihren groben Grundzügen skizziert und auch nicht annährend mit der gleichen Ausführlichkeit wie die Schema-Theorie beschrieben werden.

\subsection{Nicht-strukturelle Schema-Theorie}

Mit der nicht-strukturellen Schema-Theorie ist im Wesentlichen der Entwurf von IranNejad und Kollegen gemeint (vgl. Iran-Nejad, 1987; Iran-Nejad \& Homaifar, 1991; 
Iran-Nejad \& Homaifar, 2000; Iran-Nejad \& Winsler, 2000; vgl. auch Brewer, 2000a). ${ }^{8}$ Ausgangspunkt der Überlegungen von Iran-Nejad (1987) ist die Feststellung, dass die strukturelle, d. h. die gemeinhin gebräuchliche Schema-Theorie (z. B. Neisser, 1967; Rumelhart, 1980) sich zwar in fast allen Arbeiten auf Bartlett bezieht, aber dennoch starke Unterschiede zu den ursprünglichen Annahmen von Bartlett aufweist. Während die strukturelle Schema-Theorie von statischen, monolithischen Wissensstrukturen im Langzeitgedächtnis spricht, meinte Bartlett mit dem Begriff Schema eigentlich dynamische, sich ständig bildende und neu verändernde Muster (vgl. auch Brewer, 2000a, 2000b; Edwards \& Middleton, 1987; Ost \& Costall, 2002). In der strukturellen Schema-Theorie sind Schemata hingegen kognitive Bausteine, die gesucht, gefunden und aktiviert werden (vgl. Kap. 2). Iran-Nejad (1987) zeigt anhand mehrerer Textstellen in der gängigen schema-theoretischen Literatur diese Fehlinterpretation Bartletts und geht mit seinem Entwurf gewissermaßen „back to Bartlett“. Wie bereits an anderer Stelle ausgeführt, kritisiert Iran-Nejad (1987; vgl. auch Iran-Nejad \& Homaifar, 1991; Iran-Nejad \& Winsler, 2000) den statischen und unflexiblen Charakter der strukturellen SchemaTheorie: „It is because of the requirement of ,continuous change" in the face of constantly novel situations that the idea of schemata as pre-existing long-term memory structures becomes paradoxical“ (Iran-Nejad, 1987, S. 114). Ausgehend davon begreift Iran-Nejad (1987) Schemata als vorübergehende, funktionale (d. h. zweckmäßige) Muster. Schemata werden nicht im Langzeitgedächtnis abgespeichert oder „gelagert“, sondern sie entstehen nur für den Moment. Das kognitive Netzwerk ist für den Autor hoch plastisch, dynamisch; es ist ständig in Veränderung. Anders formuliert, das Ganze ist sprichwörtlich mehr als die Summe seiner Teile. Es besteht nicht einmal aus einzelnen, zusammensetzbaren Teilen, Bausteinen oder Schemata, es wird vielmehr ständig neu aus seinen Teilen heraus generiert (Iran-Nejad, 1987, S. 114). Iran-Nejad umgeht mit seiner Konzeption den immer wieder kritisierten Schwachpunkt der Schema-Theorie: der starren Auffassung von Informationsverarbeitungsprozessen. Eine vollends überarbeitete, empirisch zu prüfende Theorie wird aber nicht vorgelegt, so dass bis dato eher von einem Entwurf zu sprechen ist. Brewer (2000a) gibt zu bedenken, dass sich mit der nichtstrukturellen Schema-Theorie die erinnerungsstützende Funktion von Schemata nunmehr schwerer erklären lässt. Des Weiteren fordert Brewer (2000a) von den Autoren eine deutlichere Positionierung zu konnektionistischen Modellen, die bei Iran-Nejad (1987) gar nicht und bei Iran-Nejad \& Winsler (2000) nur ganz am Rande erwähnt werden. Die Überlegungen von Iran-Nejad könnten aber dennoch für die Medienwirkungsforschung wichtige Denkanstöße geben. Zwar wird das Problem der prognostischen und operationalen Präzision nicht gelöst, allerdings wird der Schema-Theorie zumindest ein validerer und - gemessen am Stand der Kognitionsforschung - modernerer Anstrich verpasst.

8 Es mag etwas verwirrend sein, warum die nicht-strukturelle Schema-Theorie nicht im obigen Kapitel unter „der Schema-Theorie“ besprochen wurde. Wenn in der Literatur von „der" Schema-Theorie die Rede ist, ist eigentlich immer die klassische, strukturelle Schema-Theorie gemeint. Die hier skizzierte nicht-strukturelle Auffassung von Schemata wurde in der Wirkungsforschung bisher nicht wahrgenommen. Auch in der Kognitionspsychologie ist die Resonanz auf den Entwurf von Iran-Nejad (1987) eher als gering einzuschätzen. Dies könnte aber auch daran liegen, dass dort schema-theoretische Argumentationen ohnehin immer seltener anzutreffen sind. 


\subsection{Konnektionistische Modelle}

Der Grundstein für konnektionistische Modelle wurde von der Parallel Distributed Processing (PDP) Research Group um die Forscher Rumelhart und McClelland (vgl. Rumelhart et al., 1986) gelegt. ${ }^{9}$ Ausgangspunkt einer parallel distributiven Verarbeitung - synonym oft auch als Konnektionismus oder künstliche neuronale Netzwerke bezeichnet - ist der Versuch, auf der Grundlage von Computersimulationen Erkenntnisse über kognitive Prozesse zu erhalten. Kognitive Prozesse werden so modelliert, dass sie dem zugrunde liegenden biologischen Vorbild weitestgehend ähnlich sind. Kurz: Es geht um eine neuronal inspirierte Modellbildung kognitiver Prozesse (vgl. Pospeschill, 2004, S. 17). Konnektionistische Modelle gehen von adaptiven informationsverarbeitenden Systemen aus, die sich aus einer Vielzahl von Verarbeitungseinheiten (units) zusammensetzen und Signale in Form von Aktivierungsmustern über gerichtete Verbindungen übertragen. Dies ist als eine grobe Analogie zum biologischen Nervensystem aufzufassen, bei dem Informationsverarbeitung durch einen Verbund von Nervenzellen realisiert wird (Pospeschill, 2004, S. 26). Vereinfacht ausgedrückt werden in einer parallel-distributiven Verarbeitung Informationen als Aktivierungsmuster einzelner units repräsentiert. Diese units sind in einem Netzwerk von Verbindungen miteinander verknüpft: „The activation of each unit is a function of the sum of the activations it received from other units, weighted by the strengths of the connections to it from each of these units“ (Eiser, Fazio, Stafford \& Prescott, 2003, S. 1223). Damit wird eine ganz andere Auffassung von Wissen, Gedächtnis und Lernen vorgelegt, als dies noch in der SchemaTheorie der Fall war (vgl. Pospeschill, 2004, S. 216-227). Nach Ansicht von Rumelhart et al. (1986; ähnlich Pospeschill, 2004) befindet sich nämlich die strukturelle SchemaTheorie in einem Dilemma: Wie kann ein Schema eine abstrakte Struktur bzw. Wissensrepräsentation sein und gleichzeitig ausreichend formbar, um für die verschiedensten Situationen zuzutreffen? Rumelhart et al. (1986) lösen diese Frage, indem sie ähnlich wie Iran-Nejad (1987) vom strukturellen Aspekt der Informationsverarbeitung Abstand nehmen: Wissen wird nicht explizit in Form von lokalen Symbolträgern wie Schemata gespeichert, sondern es entsteht gewissermaßen implizit als Aktivierungsmuster einzelner neuronaler Elemente. Beim Konnektionismus ist die Repräsentation distributiv und subsymbolisch; sie ist ferner aktiv, da sie sich über Aktivierungsmuster weiter verändern kann. Ein entscheidender Unterschied zur Schema-Theorie und damit zur so genannten symbolischen Auffassung von Kognition ist die massiv parallele, d. h. gleichzeitige Aktivität vieler units (Pospeschill, 2004, S. 28). Ferner verläuft die Informationsverarbeitung nicht nach dem Alles-oder-Nichts Prinzip: Das Informationsverarbeitungssystem muss nicht entscheiden, ob dieses oder jenes Schema herangezogen wird: „[A]ll types of cognitive representations will be found to be flexibly reconstructed in a context-sensitive way rather than retrieved from memory as they were stored like items buried in a time capsule“ (Smith, 1996, S. 901).

In einem konnektionistischen Netzwerk könnte man zwar so etwas wie ein Schema modellieren (vgl. Pospeschill, 2004, S. 226), wenn ein bestimmtes neuronales Muster mehrmals aktiviert und daher stabilisiert wird (vgl. auch Kintsch, 1998). Allerdings

9 Rumelhart galt als einer der wichtigsten Vertreter der Schema-Theorie in den 70er und frühen 80 er Jahren. In gewisser Hinsicht ist es bezeichnend für den Status der Schema-Theorie in der neueren kognitiven Psychologie, wenn sich einer ihrer prominentesten Hauptvertreter von ihr abwendet und der „alten“ Schema-Theorie ein neues Theoriegebäude gegenüberstellt. 
müsste man sich dann von einigen wichtigen Grundannahmen der Schema-Theorie verabschieden. Aus konnektionistischer Sicht wird ein Schema nicht mehr gespeichert, gelagert und abgerufen. Dessen ungeachtet wird die Schema-Theorie und der Konnektionismus fälschlicherweise immer wieder in einen Topf geworfen. Dass damit fundamentale Unterschiede in Bezug auf Wissen, Gedächtnis und Lernen verwischt werden (vgl. Pospeschill, 2004, S. 216-227), wird leider übersehen. Es ist zu betonen, dass die konnektionistische Sicht nicht mehr viel - und damit viel weniger als noch die nichtstrukturelle Schema-Theorie - mit der (strukturellen) Schema-Theorie zu tun hat (für die Unterschiede zwischen beiden vgl. Pospeschill, 2004; Reynolds, Sinatra \& Jetton, 1996; Smith, 1996; Wirth, 1997).

Worin könnte nun aber der Vorteil von konnektionistischen Modellen im Vergleich zur Schema-Theorie liegen? Zunächst einmal zeichnen konnektionistische Modelle generell ein viel flexibleres, umfassenderes und damit adäquateres Bild von Informationsverarbeitungsprozessen (Nassaji, 2002; Smith, 1996; Wirth, 1997). Zudem sind sie erklärungskräftiger, wenn es um das Lernen von neuen Informationen geht (Smolensky, 1986, S. 261). Auch die in der Schema-Theorie vernachlässigten affektiven Komponenten können berücksichtigt bzw. modelliert werden. Im Vergleich zur Schema-Theorie erhöht sich damit zwar der erklärende, nicht aber notwendigerweise der prognostische Gehalt. Durch den Einsatz von Computersimulationen sieht sich der Konnektionismus mit anderen Problemen und anderen Theorieanforderungen konfrontiert als die Schema-Theorie. Der Konnektionismus ist ein Tool, mit dem komplexe Wirkungsprozesse simuliert und modelliert werden könnten. Ziel von konnektionistischen Modellierungen könnte es sein, zu angemesseneren Theorien über kognitive Funktionen zu gelangen (Seidenberg, 1993, S. 228). Welche Rolle dem Konnektionismus dabei zukommt und zukommen kann, ist aber derzeit heftig umstritten (Green, 2001; McCloskey, 1991; Seidenberg, 1993). In der Kommunikationswissenschaft finden sich bis dato nur vereinzelte Anwendungen der konnektionistischen Denkweise (Vorderer \& Weber, 2003; Weber, 2001, Wirth, 1997), so dass die Brauchbarkeit für die Modellierung von Wirkungsprozessen noch nicht abzuschätzen ist. Allerdings geht die Komplexität der Sachverhalte, die mittlerweile mit konnektionistischen Netzwerken simuliert werden können, weit über einfache Modellierungen (z. B. Worterkennung) hinaus, und dies lässt viel versprechende Anwendungsmöglichkeiten erwarten (vgl. u. a. Eiser et al. 2003; Seidenberg, 1993; Siegle \& Hasselmo, 2002; Smith, 1996).

\subsection{Einstellungsforschung}

Selbst in den "goldenen“ 70er und 80er Jahren der Schema-Theorie wurde immer wieder die Frage aufgeworfen, ob Schemata im Grunde nichts anderes seien als die kognitiven Komponenten von Einstellungen bzw. Einstellungen in einem anderen Gewand (vgl. Fiske \& Linville, 1980; Wildavsky, 1987). In der Tat erinnert die schon im Jahr 1935 von Allport vorgenommene Charakterisierung von Einstellungen sehr stark an die Schema-Theorie: „Attitudes determine for each individual what he will see and hear, what he will think and what he will do. To borrow a phrase from William James, they, engender meaning upon the world““ (Allport, 1935, S. 806). So ist es wenig verblüffend, wenn die in der Sozialpsychologie entwickelten Einstellungstheorien die gleichen Phänomene erklären können wie die vornehmlich in der Kognitionspsychologie vorangetriebene Schema-Theorie: Einstellungen beeinflussen ebenso wie Schemata die Informationsverarbeitung in Bezug auf Aufmerksamkeit, Enkodierung, Elaboration und Gedächtnis (Eagly \& Chaiken, 1998; Ostrom, Skowronski et al., 1994; Roskos-Ewoldsen, 1997). 
Der größte Vorteil im Vergleich zur Schema-Theorie sind jedoch die elaborierten und ausführlich erforschten Annahmen zu Einstellungsänderungen, wie sie beispielsweise im elaboration-likelihood-model (ELM, vgl. Petty \& Wegener, 1999) und im heuristicsystematic-model (HSM, vgl. Chen \& Chaiken, 1999) getroffen werden. Diese Modelle erlauben nicht nur präzise Aussagen über die Bedingungen von Einstellungsänderungen, sondern sie stellen sich auch einer dezidierten empirischen Prüfung (vgl. Petty, Cacioppo, Strathman \& Priester, 1994). Zudem ermöglicht beispielsweise das HSM eine sehr elegante Einbindung von Prozesskonstrukten wie beispielsweise Heuristiken (Chen \& Chaiken, 1999). Ein weiteres gewichtiges Argument ist, dass die Einstellungsforschung explizit den Zusammenhang zwischen Kognition und Affekt modelliert: Die zentrale Annahme der Schema-Theorie, dass kognitive Komponenten losgelöst von affektiven im Langzeitgedächtnis abgespeichert werden, gilt in der neueren Einstellungsforschung als widerlegt: Die Informationen über ein Objekt - beispielsweise zu einem politischen Thema - sind affektiv aufgeladen, und diese affektiven Komponenten werden bei der Stimuluswahrnehmung mitaktiviert. Man spricht in diesem Zusammenhang auch von heißer Kognition (hot cognition): „[E]motional evaluation is stored with the concept - that is, political terms are indeed ,hot"“ (Morris et al., 2003, S. 742). Dies macht einen entscheidenden Mehrwert gegenüber der Schema-Theorie aus. Auch gilt die Operationalisierung von Einstellungen als weitaus fundierter als die von Schemata (vgl. Ostrom, Bond et al., 1994). Nicht zuletzt liegen in der Einstellungsliteratur elaborierte Konzepte zur Einstellungsstärke, Einstellungsresistenz und Einstellungsstruktur vor (vgl. einführend Ostrom, Skowronski et al., 1994; Petty, Priester \& Briñol, 2002; Wegener, Petty, Smoak \& Fabrigar, 2004). Auch über diese Aspekte weiß die Schema-Theorie wenig zu sagen.

Die Kommunikationswissenschaft hat die Erforschung von Einstellungen und Einstellungsänderungen in den letzten 20 Jahren eher vernachlässigt (Roskos-Ewoldsen, 1997). Verantwortlich für diese Zurückhaltung ist sicherlich die weit verbreitete Auffassung, dass Einstellungen änderungsimmune Prädispositionen sind, die durch die Medienberichterstattung nicht zu beeinflussen seien. Neuere Arbeiten verabschieden sich aber zunehmend von einem statischen Einstellungsbegriff und begreifen Einstellungen auch als temporäre Konstruktionen: „[M]any political attitudes appear to be constructed on the spot from the interaction between currently active knowledge structures and information from the environment" (Taber et al., 2001, S. 201; vgl. auch Bassili \& Roy, 1998; Fazio, 2001; Lieberman, Schreiber \& Ochsner, 2003; Morris et al., 2003; RoskosEwoldsen, 1997; Wilson \& Hodges, 1992). Somit lässt sich auch mit Smith (1996) eine Anbindung der Einstellungsforschung an konnektionistische Prinzipien erkennen. Möglicherweise wurde das Potenzial der Einstellungsforschung für die Medienwirkungsforschung noch nicht richtig ausgeschöpft. So wäre es durchaus denkbar, dass so manche Wirkungsstudie, die schema-theoretische Erklärungen heranzieht, ebenso mit Einstellungstheorien argumentieren könnte. Ob es schließlich sinnvoll ist, Medienwirkungsprozesse als rein kognitiv aufzufassen, d. h. gänzlich losgelöst von affektiven Prozessen zu betrachten, mag bezweifelt werden.

\section{Zusammenfassung \& Ausblick}

„The use of the term schema is widespread, vague, and not always overladen with meaning“ (Brown, 1979, S. 231). Diese schon vor 25 Jahren verfasste Beurteilung der Schema-Theorie - so das Fazit dieses Aufsatzes - besitzt noch immer ihre Gültigkeit. Wie aufgezeigt werden konnte, ist die Schema-Theorie als Erklärungsmodell für Informati- 
onsverarbeitungsprozesse zugleich weit verbreitet, aber auch sehr umstritten. Der Erklärungswert der Schema-Theorie für Wirkungsprozesse lässt sich an drei Punkten festmachen: Die Theorie versucht zum Ersten zu erklären, wie die Rezipienten bestehendes Wissen strukturieren, um die Fülle der medial vermittelten Informationen zu meistern. Zum Zweiten gibt die Schema-Theorie Aufschluss darüber, wie der Prozess der Informationsaufnahme und des Informationsabrufes erfolgt: Medial vermittelte Informationen werden nicht eins zu eins übernommen, sondern in bereits bestehende Schemata integriert. Zum Dritten geht die Schema-Theorie auf aktive Konstruktionsprozesse der Rezipienten ein. Schemata ermöglichen gewissermaßen, mehr aus den Informationen zu machen. In der Medienwirkungsforschung hat sich die Schema-Theorie mit dieser Erklärungskraft als heuristisch erfolgreich erwiesen. In vielen Forschungsgebieten wurden durch die Schema-Theorie Informationsverarbeitungsprozesse in den Vordergrund gerückt und empirische Resultate kognitionspsychologisch untermauert.

Trotz dieser positiven Impulse erweist sich der Nutzen der Schema-Theorie für die Wirkungsforschung aus hauptsächlich drei Gründen als begrenzt. Erstens gibt es Probleme bei der Operationalisierung von Schemata. Die eingesetzten Techniken sind vor allem in Hinsicht auf die recht willkürliche Ableitung und Interpretation der Schemata zu kritisieren. Das Problem ist, dass jegliche Ergebnisse als Schemata interpretiert werden können, ohne dass klar ist, ab wann denn von einem Schema zu sprechen ist. Ein Grund für die recht schwammigen empirischen Umsetzungen ist sicherlich in der Definition von Schemata auszumachen (Taylor \& Crocker, 1981). Das ist das zweite Problem der Schema-Theorie. Nach Williams (1999) ist dieses Phänomen vielen abstrakten kognitiven Konstrukten gemein, die ähnlich wie die Schema-Theorie eine hohe intuitive Plausibilität haben: „[They] have so much surface appeal that we embrace them even before clearly defining them" (Williams, 1999, S. 412). Noch schwer wiegender ist aber das dritte Problem der Schema-Theorie: ihr mangelnder prognostischer Wert. Die Schema-Theorie zeichnet aufgrund ihrer zu statischen Konzeption ein vereinfachtes Bild von Informationsverarbeitungsprozessen. In der modernen Kognitionspsychologie herrscht schon lange Einigkeit darüber, dass keine vorgefertigten „Bausteine der Kognition“ (Rumelhart, 1980, S. 33) aus dem Langzeitgedächtnis abgerufen und über die Stimuli gestülpt werden. Zudem können Veränderungen von Schemata sowie das Zusammenspiel von Kognition und Affekt nur unzureichend schema-theoretisch erklärt werden. Ebenso weiß die Schema-Theorie keine Antwort auf die in vielen Studien berichtete a-schematische Informationsverarbeitung bei detailgetreuen bzw. exakten Erinnerungen (Wirth, 1997, S. 127). Hinzu kommt die Flexibilität der Theorie in Bezug auf die empirischen Ergebnisse. Damit ist gemeint, dass sie einerseits zu viel und andererseits zu wenig erklärt: $\mathrm{Zu}$ viel, weil mit der Schema-Theorie nahezu jedes empirische Ergebnis gedeutet werden kann - zu wenig, da sie keine wirklich exakten Vorhersagen über Wirkungsprozesse erlaubt. Das einfache Referieren auf „Wissensstrukturen“, die „unsere“ Wahrnehmung von Medienstimuli steuern, ist für eine prozessorientierte Medienwirkungsforschung nicht ausreichend.

Wie kann diesen Kritikpunkten in zukünftigen Arbeiten begegnet werden? Wenn man an der Schema-Theorie festhalten will, scheint es zuallererst vonnöten, stärker auf die definitorische Präzision zu achten. Hierbei sollte sich vor allem um eine operationale Definition bemüht werden (vgl. Williams, 1999). Zudem würde eine Beachtung der nicht-strukturellen Schema-Theorie auch zu einer flexibleren Auffassung von Informationsverarbeitungsprozessen führen, ohne dabei auf den Schema-Begriff verzichten zu müssen. Außerdem gilt es, der Operationalisierung von Schemata mehr Augenmerk beizumessen. Hierzu lassen sich beispielsweise bei Scheufele (2004) erste Hinweise finden. 
Dennoch bleibt fraglich, ob ein Ausbau bzw. eine Weiterentwicklung der SchemaTheorie - wie dies immer wieder von kommunikationswissenschaftlicher Seite gefordert wird (vgl. Wicks, 1992; Schenk, 2002, S. 296) - auf Dauer für die Medienwirkungsforschung Gewinn bringend ist. Die Schema-Theorie kann sich längerfristig nur dann als fruchtbar erweisen, wenn sie einen adäquaten, widerspruchsfreien und präzisen Erklärungswert liefert. Ob dies aber der Fall ist bzw. der Fall sein wird, darf bezweifelt werden. Die Schema-Theorie ermöglicht zwar durch ihre Fokussierung von Informationsverarbeitungsprozessen einen Blick in die „Black Box“ - dieser ist aber eher als „unscharf“ zu bezeichnen.

Es lässt sich zumindest die Empfehlung ableiten, dass immer dann auf eine schematheoretische Argumentation verzichtet werden sollte, wenn konkretere, aussagekräftigere Konzepte zur Verfügung stehen. Welche das sein könnten, hängt sicherlich vom jeweiligen Forschungsgegenstand ab. Die hier skizzierten Alternativen zur Schema-Theorie sind möglicherweise ein Anfang. Konnektionistische Ansätze kommen der Komplexität und Flexibilität menschlichen Denkens weitaus näher, als dies die Schema-Theorie tut. Einstellungstheorien haben darüber hinaus Vorteile in Bezug auf die standardmäßige Operationalisierung und die Erklärung von Einstellungsänderungen. Beide erlauben Vorhersagen über das Zusammenspiel von Kognition und Affekt in Medienwirkungsprozessen. Insgesamt könnte also eine stärkere Orientierung an aktuelleren kognitionsund sozialpsychologischen Konzepten zu einem vertieften Verständnis von Wirkungsprozessen führen. Dass andere Disziplinen keine Fertigrezeptur für kommunikationswissenschaftliche Fragestellungen bereithalten, soll damit nicht bestritten werden. Sicherlich bringen die Alternativen zur Schema-Theorie sogar neue Fragen und möglicherweise auch neue Probleme mit sich, für die erst Antworten und Lösungen erarbeitet werden müssen. Auf diesem, vielleicht auch etwas steinigen Weg bleibt dennoch die Gewissheit, dass es besser ist, neue Fragen und Probleme anzugehen, statt weiterhin mit veralteten Modellen zu arbeiten, mit denen man ohnehin nicht recht zufrieden ist.

\section{Literatur}

Alba, J. W. \& Hasher, L. (1983). Is memory schematic? Psychological Bulletin, 93, 203-231.

Allport, G. W. (1935). Attitudes. In G. Murchison (Hrsg.), Handbook of social psychology (S. 798-844). Worcester, MA: Clark University Press.

Anderson, R. C. (1977). The notion of schemata and the educational enterprise: General discussion of the conference. In R. C. Anderson, R. J. Spiro \& W. E. Montague (Hrsg.), Schooling and the acquisition of knowledge (S. 415-431). Hillsdale, NJ: Erlbaum.

Bartlett, F. C. (1932). Remembering. Studies in experimental social psychology. London: Cambridge University Press.

Bassili, J. N. \& Roy, J.-P. (1998). On the representation of strong and weak attitudes about policy in memory. Political Psychology, 19(4), 669-681.

Bilandzic, H. (1999). Psychische Prozesse bei der selektiven Fernsehnutzung. Beschreibung und Erklärung von Programmentscheidungen mit der Methode des lauten Denkens. In U. Hasebrink \& P. Rössler (Hrsg.), Publikumsbindungen: Medienrezeption zwischen Individualisierung und Integration (S. 89-110). München: Fischer.

Biocca, F. (1991). Viewers' mental models of political messages: Toward a theory of the semantic processing of television. In F. Biocca (Hrsg.), Television and political advertising (S. 27-89). Hillsdale, NJ: Erlbaum.

Bolland, J. M. (1985). The structure of political cognition: A new approach to its meaning and measurment. Political Behavior, 7(3), 248-265.

Brewer, W. F. (2000a). Bartlett, functionalism, and modern Schema Theories. The Journal of Mind and Behavior, 21(1-2), 37-44. 
Brewer, W. F. (2000b). Bartlett's concept of the schema and its impact on theories of knowledge representation in contemporary cognitive psychology. In A. Saito (Hrsg.), Bartlett, culture and cognition (S. 69-89). Philadelphia: Psychology Press.

Brosius, H.-B. (1991). Schema-Theorie - ein brauchbarer Ansatz in der Wirkungsforschung? Publizistik, 36, 285-297.

Brown, A. L. (1979). Theories of memory and problems of development: Activity, growth, and knowledge. In L. S. Cermak \& F. I. M. Craik (Hrsg.), Levels of processing in human memory (S. 225-258). Hillsdale, NJ: Erlbaum.

Chen, S. \& Chaiken, S. (1999). The heuristic-systematic model in its broader context. In S. Chaiken \& Y. Trope (Hrsg.), Dual-process theories in social psychology (S. 73-97). New York, London: Guilford.

Coleman, R. (2003). Race and ethical reasoning: The importance of race to journalistic decision making. Journalism \& Mass Communication Quarterly, 80(2), 295-310.

Conover, P. J. \& Feldman, S. (1984). How people organize the political world: a schematic model. American Journal of Political Science, 28, 95-126.

Crockett, W. H. (1987). Schemas, affect, and communication. In L. Donohew, H. E. Sypher \& E. T. Higgins (Hrsg.), Communication, Social Cognition, and Affect (S. 33-49). Hillsdale, NJ: Erlbaum.

Cromrey, A. L. (1988). Factor-analytic methods of scale development in Personality and Clinical Psychology. Journal of Consulting and Clinical Psychology, 56, 754-761.

Eagly, H. \& Chaiken, S. (1998). Attitude structure and function. In D. T. Gilbert, S. T. Fiske \& G. Lindzey (Hrsg.), Handbook of Social Psychology (S. 269-281). Boston: McGraw-Hill.

Edwards, D. \& Middleton, D. (1987). Conversation and remembering: Bartlett revisited. Applied Cognitive Psychology, 1, 77-92.

Eichhorn, W. (1996). Agenda-Setting-Prozesse. Eine theoretische Analyse individueller und gesellschaftlicher Themenstrukturierung. München: Fischer.

Eiser, J. R., Fazio, R. H., Stafford, T. \& Prescott, T. J. (2003). Connectionist simulation of attitude learning: Asymetries in the acquisition of positive and negative evaluations. Personality and Social Psychology Bulletin, 29(10), 1221-1235.

Entman, R. M. (1989). How the media affect what people think: An information processing approach. The Journal of Politics, 51(2), 347-370.

Eveland, W. P., Marton, K. \& Seo, M. (2004). Moving beyond „just the facts“. The influence of online news on the content and structure of public affairs knowledge. Communication Research, 31(1), 82-108.

Eysenck, M. W. \& Keane, M. T. (2002). Cognitive psychology: a student's handbook. Hove: Psychology Press.

Fazio, R. H. (2001). The automatic activation of associated evaluations: an overview. Cognition and Emotion, 15(2), 115-141.

Fiske, S. T. \& Linville, P. (1980). What does the schema concept buy us? Personality and Social Psychology Bulletin, 6(4), 542-557.

Fiske, S. T. \& Taylor, S. E. (1991). Social Cognition. New York: MacGraw.

Fredin, E. S. \& Tabaczynski, T. (1993). Media schemata, information-processing strategies, and audience assessment of the informational value of quotes and background in local news. Journalism E Mass Communication Quarterly, 70(4), 801-814.

Früh, W. (1996). Realitätsvermittlung durch Massenmedien. Die permanente Transformation der Wirklichkeit. Opladen: Westdeutscher Verlag.

Garramone, G. M., Steele, M. E. \& Pinkleton, B. (1991). The role of cognitive schemata in determining candidate characteristic effects. In F. Biocca (Hrsg.), Television and political advertising (S. 311-328). Hillsdale, NJ: Erlbaum.

Gehrau, V. (2003). (Film-)Genres und die Reduktion von Unsicherheit. Medien E Kommunikationswissenschaft, 51, 213-231.

Graber. D. A. (1988). Processing the news: How people tame the information tide [2nd ed.]. Lanham, MD: University Press of America.

Graesser, A. C., \& Nakamura, G. V. (1982). The impact of a schema on comprehension and 
memory. In G. H. Bower (Hrsg.), The psychology of learning and motivation (Vol. 16) (S. 59-109). New York: Academic Press

Green, C. D. (2001). Scientific models, connectionist networks, and cognitive science. Theory $\mathcal{E}$ Psychology, 11(1), 97-117.

Grimes, T. \& Drechsel, R. (1996). Word-picture juxtaposition, schemata, and defamation in television news. Journalism \& Mass Communication Quarterly, 73(1), 169-180.

Hamill, R., Lodge, M. \& Blake, F. (1985). The breadth, depth, and utility of class, partisan, and ideological schemata. American Journal of Political Science, 29(4), 850-870.

Hannover, B., Mauch, M. \& Leffelsend, S. (2004). Sozialpsychologische Grundlagen. In R. Mangold, P. Vorderer \& G. Bente (Hrsg.), Lehrbuch der Medienpsychologie (S. 175-197). Göttingen: Hogrefe.

Herrmann, T. (1982). Über begriffliche Schwächen kognitivistischer Kognitionstheorien: Begriffsinflation und Akteur-System-Kontamination. Sprache und Kognition, 1, 3-14.

Höijer, B. (1992). Reception of television narration as a socio-cognitive process: A schema-theoretical outline. Poetics, 21, 283-304.

Iran-Nejad, A. \& Homaifar, A. (1991). Assoziative und nicht-assoziative Theorien des verteilten Lernen und Erinnerns. In S. J. Schmidt (Hrsg.), Gedächtnis: Probleme und Perspektiven der interdisziplinären Gedächtnisforschung (S. 206-249). Frankfurt am Main: Suhrkamp.

Iran-Nejad, A. \& Homaifar, A. (2000). The nature of distributed learning and remembering. Journal of Mind E Behavior, 21, 153-184.

Iran-Nejad, A. \& Winsler, A. (2000). Bartlett's schema theory and modern accounts of learning and remembering. Journal of Mind E Behavior, 21, 5-35.

Iran-Nejad, A. (1987). The schema: a long term memory structure or a transient structural phenomen. In R. J. Tierney, P. L. Anders \& J. N. Mitchell (Hrsg.), Reading comprehension (S. 109-127). Hillsdale, NJ: Erlbaum.

Kardash, C. M., Royer, J. M. \& Greene, B. A. (1988). Effects of schemata on both encoding and retrieval of information from prose. Journal of Educational Psychology, 80(3), 324-329.

Kepplinger, H. M. \& Daschmann, G. (1997). Today's news - tomorrow's context: A dynamic model of news processing. Journal of Broadcasting E Electronic Media, 41, 548-565.

Kintsch, W. (1998). The representation of knowledge in minds and machines. International Journal of Psychology, 33(6), 411-420.

Kuklinski, J. H., Luskin, R. C. \& Bolland, J. (1991). Where is the Schema? Going beyond the „S“ word in political psychology. American Political Science Review, 85, 1341-1356.

Lieberman, M. D., Schreiber, D. \& Ochsner, K. N. (2003). Is political cognition like riding a bicycle? How Cognitive Neuroscience can inform research on political thinking. Political Psychology, 24(4), 881-704.

Locksley, A., Stangor, C., Hepburn, C., Grosovsky, E. \& Hochstrasser, M. (1984). The ambiguity of recognition memory tests of schema theories. Cognitive Psychology, 16, 421-448.

Markus, H. (1977). Self-schemata and processing information about the self. Journal of Personality and Social Psychology, 35(2), 63-78.

McCloskey, M. (1991). Networks and theories: The place of connectionism in cognitive science. Psychological Science, 2(6), 387-395.

McGraw, K. (2000). Contributions of the cognitive approach to Political Psychology. Political Psychology, 21(4), 805-832.

Miller, A. H., Wattenberg, M. P. \& Malanchuk, O. (1986). Schematic assessments of presidential candidates. American Political Science Review, 80(2), 521-540.

Minsky, M. (1975). A framework for representing knowledge. In. P. H. Winston (Hrsg.), The psychology of computer vision (S. 211-277). New York: McGraw-Hill.

Morris, J. P., Squires, N. K., Taber, C. S. \& Lodge, M. (2003). Activation of political attitudes: A psychophysiological examination of the hot cognition hypothesis. Political Psychology, 24(4), 727-745.

Nassaji, H. (2002). Schema theory and knowledge based processes in second language reading comprehension: A need for alternative perspectives. Language Reading, 52(2), 439-481. 
Neisser, U. (1967). Cognitive Psychology. New York: Appleton.

Ohler, P. \& Nieding, G. (1996). Cognitive modeling of suspense-inducing structures in narrative films. In P. Vorderer, H. J. Wulff \& M. Friedrichsen (Hrsg.), Suspense: conceptualisations theoretical analysis, and empirical explorations (S. 129-147). Mahwah, NJ: Erlbaum.

Ost, J. \& Costall, A. (2002). Misremembering Bartlett: A study in serial reproduction. British Journal of Psychology, 93, 243-255.

Ostrom, T. M., Bond, C. F., Krosnick, J. A. \& Sedikides, C. (1994). Attitude scales. How we measure the unmeasureable. In S. Shavitt \& T. C. Brock (Hrsg.), Persuasion: Psychological insights and perspectives (S. 15-42), Boston, MA: Allyn and Bacon.

Ostrom, T. M., Skowronski, J. J. \& Nowak, A. (1994). The cognitive foundations of attitudes: It's a wonderful construct. In P. G. Devine, D. L. Hamilton \& T. M. Ostrom (Hrsg.), Social Cognition: Impact on Social Psychology (S. 195-258). San Diego: Academic Press.

Pan, Z. \& Kosicki, G. M. (1993). Framing analysis: an approach to news discourse. Political Communication, 10, 55-76.

Petty, R. E. \& Wegener, D. T. (1999). The elaboration-likelihood model: Current status and controversies. In Chaiken, S. \& Trope, Y. (Hrsg.), Dual-process theories in social psychology (S. 41-72). New York, London: Guilford.

Petty, R. E., Cacioppo, J. T., Strathman, A. J. \& Priester, J. R. (1994). To think or not to think. Exploring two routes to persuasion. In S. Shavitt \& T. C. Brock (Hrsg.), Persuasion: Psychological insights and perspectives (S. 113-147). Boston, MA: Allyn and Bacon.

Petty, R. E., Priester, J. R. \& Briñol, P. (2002). Mass media attitude change: Implications of the Elaboration Liklihood Model of persuasion. In J. Bryant \& D. Zillmann (Hrsg.), Media effects. Advances in theory and research (S. 155-198). Mahwah, NJ: Erlbaum.

Pospeschill, M. (2004). Konnektionismus und Kognition. Eine Einführung. Stuttgart: Kohlhammer.

Prabu, D. (1996). Role of imagery in recall of deviant news. Journalism E Mass Communication Quarterly, 73(4), 804-820.

Reynolds, R. E., Sinatra, G. M. \& Jetton, T. L. (1996). Views of knowledge acquisition and representation: A continuum from experience centered to mind centered. Educational Psychologist, 31(2), 93-104.

Rhee, J. \& Capella, J. N. (1997). The role of political sophistication in learning from news: Measuring schema development. Communication Research, 24(3), 197-233.

Roskos-Ewoldsen, D. R. (1997). Attitude accessibility and persuasion: Review and a transactive model. In B. R. Burleson \& A. W. Kunkel (Hrsg.), Communication Yearbook (S. 185-225), Thousand Oaks: Sage.

Rössler, P. (1997). Agenda-Setting. Theoretische Annabmen und empirische Evidenzen einer Medienwirkungshypothese. Opladen: Westdeutscher Verlag.

Ruhrmann, G. (1989). Rezipient und Nachricht. Struktur und Prozeß der Nachrichtenkonstruktion. Opladen: Westdeutscher Verlag.

Rumelhart, D. E. \& Norman, D. A. (1978). Accretion, tuning, and restructuring: Three models of learning. In J. W. Cotton \& R. L. Klatzky (Hrsg.), Semantic factors in cognition (S. 37-53). Hillsdale, NJ: Erlbaum.

Rumelhart, D. E. \& Ortony, A. (1977). The representation of knowledge in memory. In R. C. Anderson, R. J. Spiro \& W. E. Montague (Hrsg.), Schooling and the acquisition of knowledge (S. 99-135). Hillsdale, NJ: Erlbaum.

Rumelhart, D. E. (1980). Schemata: The building blocks of cognition. In R. J. Spiro, B. C. Bruce \& W. F. Brewer (Hrsg.), Theoretical issues in reading comprehension: perspectives from cognitive psychology (S. 33-58). Hillsdale, NJ: Erlbaum.

Rumelhart, D. E., Smolensky, P., McClelland, J. L. \& Hinton, G. E. (1986). Schemata and sequential thought processes in PDP models. In J. L. McClelland, D. E. Rumelhart, and the PDP Research Group, Parallel distributed processing. Explorations in the microstructure of cognition. Volume 2: Psychological and Biological Models (S. 7-57). Cambridge, MA: MIT Press.

Sadoski, M., Paivio, A. \& Goetz, E. T. (1991). A critique of schema theory in reading and a dual coding alternative. Reading Research Quarterly, 26, 463-484.

Schenk, M. (2002). Medienwirkungsforschung. Tübingen: Mohr. 
Scheufele, B. (2003). Frames - Framing - Framing-Effekte. Theoretische und methodische Grundlegung des Framing-Ansatzes sowie empirische Befunde zur Nachrichtenproduktion. Wiesbaden: Westdeutscher Verlag.

Scheufele, B. (2004). Framing-Effekte auf dem Prüfstand. Eine theoretische, methodische und empirische Auseinandersetzung mit der Wirkungsperspektive des Framing-Ansatzes. Medien $\mathcal{E}$ Kommunikationswissenschaft, 52(1), 30-55.

Schwan, S. (1995). „Love or crime or something else?“ Schematische Wissensstrukturen und Filmrezeption. Rundfunk und Fernsehen, 43(1), 26-40.

Schwarz, N. (1985). Theorien konzeptgesteuerter Informationsverarbeitung in der Sozialpsychologie. In D. Frey \& M. Irle (Hrsg.), Theorien der Sozialpsychologie. [Bd. 3: Motivations- und Informationsverarbeitungstheorien] (S. 269-291). Bern: Hans Huber.

Seidenberg, M. S. (1993). Connectionist models and cognitive theory. Psychological Science, 4(4), 228-235.

Shen, F. (2004). Chronic accessibility and individual cognitions: Examining the effects of message frames in political advertisements. Journal of Communication, 54(1), 123-137.

Siegle, G. J. \& Hasselmo, M. E. (2002). Using connectionist models to guide assessment of psychological disorder. Psychological Assessment, 14(3), 263-278.

Smith, E. R. (1996). What do connectionism and Social Psychology offer each other? Journal of Personality and Social Psychology, 70(5), 893-912.

Smolensky, P. (1986). Information processing on dynamical systems: foundations of harmony theory. In D. E. Rumelhart, J. L. McClelland, and the PDP Research Group, Parallel distributed processing. Explorations in the microstructure of cognition. Volume 1: Foundations (S. 194-281). Cambridge, MA: MIT Press.

Taber, C. S., Lodge, M. \& Glathar, J. (2001). The motivated construction of political judgements. In J. H. Kuklinski (Hrsg.), Citizens and politics. Perspectives from Political Psychology (S. 198-226). Cambridge: Cambridge University Press.

Tannen, D. \& Wallatt, C. (1993). Interactive frames and knowledge schemas in interaction: Examples from a medical examination/Interview. In D. Tannen (Hrsg.), Framing in discourse (S. 57-76.). Oxford: Oxford University Press.

Taylor, S. E. \& Crocker, J. (1981). Schematic bases of social information processing. In E. T. Higgins, C. P. Herman \& M. P. Zanna (Hrsg.), Social Cognition: The Ontario Symposium on Personality and Social Psychology, Vol. 1. (S. 89-134). Hillsdale, NJ: Erlbaum.

Thorndyke, P. W. \& Yekovich, F. R. (1980). A critique of schema-based theories of human story memory. Poetics, 9, 23-49.

Vorderer, P. \& Weber, R. (2003). Unterhaltung als kommunikationswissenschaftliches Problem: Ansätze einer konnektionistischen Modellierung. In W. Früh \& H.-J. Stiehler (Hrsg.), Theorie der Unterhaltung. Ein interdisziplinärer Diskurs (S. 136-159). Köln: Halem.

Warlaumont, H. G. (1997). Appropriating reality: Consumers' perceptions of schema-inconsistent advertising. Journalism \& Mass Communication Quarterly, 74(1), 39-54.

Weber R. \& Crocker J. (1983). Cognitive processes in the revision of stereotypic beliefs. Journal of Personality and Social Psychology, 45(5), 961-977.

Weber, R. (2001). Datenanalyse mittels neuronaler Netze am Beispiel des Publikumserfolgs von Spielfilmen. Zeitschrift für Medienpsychologie, 13(4), 164-176.

Wegener, D. T., Petty, R. E., Smoak, N. D. \& Fabrigar, L. R. (2004). Multiple routes to resisting attitude change. In E. S. Knowles \& J. A. Linn (Hrsg.), Resistance and persuasion (S. 13-38). Mahwah, NJ: Erlbaum.

Wicks, R. H. (1992). Schema Theory and the measurement in mass communication research: Theoretical and methodological issues in news information processing. In S. A. Deetz (Hrsg.), Communication Yearbook (S. 115-145). Newbury Park: Sage.

Wicks, R. H. (1995). Remembering the news: Effects of medium and message discrepancy on news recall over time. Journalism \& Mass Communication Quarterly, 72(3), 666-681.

Wicks, R. H., \& Drew, D. G. (1991). Learning from the news: Effects of message consistency and medium recall and medium recall on inference making. Journalism Quarterly, 68(1-2), $155-164$. 
Wilcox, C. \& Williams, L. (1990). Taking stock of schema theory. Social Science Journal, 27, 373-394.

Wildavsky, A. (1987). Choosing preferences by constructing institutions: A cultural theory of preference formation. American Political Science Review, 81(1), 3-22.

Williams, R. L. (1999). Operational definitions and assessment of higher-order cognitive constructs. Educational Psychology, 11(4), 411-427.

Wilson, T. D. \& Hodges, S. D. (1992). Attitudes as temporary constructions. In L. L. Martin \& A. Tesser (Hrsg.), The construction of social judgements (S. 37-65). Hillsdale, NJ: Erlbaum.

Winterhoff-Spurk, P. (1989). Fernsehen und Weltwissen. Der Einfluß von Medien auf Zeit-, Raumund Personenschemata. Opladen: Westdeutscher Verlag.

Wirth, W. (1997). Von der Information zum Wissen. Die Rolle der Rezeption für die Entstehung von Wissensunterschieden. Ein Beitrag zur Wissenskluftforschung. Opladen: Westdeutscher Verlag.

Wolling, J., Wünsch, C. \& Gehrau, V. (1998). Was ich nicht weiß, macht mich nicht heiß? Eine Agenda-Setting-Untersuchung aus schema-theoretischer Perspektive. Rundfunk und Fernsehen, 46(14), 447-462.

Wynn, V. E. \& Logie, R. H. (1998). The veracity of long-term memories - Did Bartlett get it right? Applied Cognitive Psychology, 12, 1-20.

Zillmann, D. \& Brosius, H.-B. (2000). Exemplification in communication: The influence of case reports on the perception of issues. Mahwah, NJ: Erlbaum.

Zuroff, D. C. (1989). Judgments of frequency of social stimuli: How schematic is person memory? Journal of Personality and Social Psychology, 56 (6), 890-898. 